\title{
Measurement of the mass and lifetime of the $\Omega_{b}^{-}$baryon
}

\author{
R. Aaij et al.* \\ (LHCb Collaboration) \\ (Received 7 April 2016; published 19 May 2016)
}

\begin{abstract}
A proton-proton collision data sample, corresponding to an integrated luminosity of $3 \mathrm{fb}^{-1}$ collected by LHCb at $\sqrt{s}=7$ and $8 \mathrm{TeV}$, is used to reconstruct $63 \pm 9 \Omega_{b}^{-} \rightarrow \Omega_{c}^{0} \pi^{-}, \Omega_{c}^{0} \rightarrow p K^{-} K^{-} \pi^{+}$decays. Using the $\Xi_{b}^{-} \rightarrow \Xi_{c}^{0} \pi^{-}, \Xi_{c}^{0} \rightarrow p K^{-} K^{-} \pi^{+}$decay mode for calibration, the lifetime ratio and the absolute lifetime of the $\Omega_{b}^{-}$baryon are measured to be $\tau_{\Omega_{b}^{-}} / \tau_{\Xi_{b}^{-}}=1.11 \pm 0.16 \pm 0.03, \tau_{\Omega_{b}^{-}}=1.78 \pm 0.26 \pm 0.05 \pm 0.06 \mathrm{ps}$, where the uncertainties are statistical, systematic and from the calibration mode (for $\tau_{\Omega_{b}^{-}}$only). A measurement is also made of the mass difference, $m_{\Omega_{b}^{-}}-m_{\Xi_{b}^{-}}$, and the corresponding $\Omega_{b}^{-}$mass, which yields $m_{\Omega_{b}^{-}}-m_{\Xi_{b}^{-}}=247.4 \pm 3.2 \pm 0.5 \mathrm{MeV} / c^{2}, m_{\Omega_{b}^{-}}=6045.1 \pm 3.2 \pm 0.5 \pm 0.6 \mathrm{MeV} / c^{2}$. These results are consistent with previous measurements.
\end{abstract}

DOI: 10.1103/PhysRevD.93.092007

\section{INTRODUCTION}

Measurements of the lifetimes of beauty baryons provide an important test of Heavy Quark Effective Theory (HQET) [1-8], in which it is predicted that the decay width is dominated by the weak decay of the heavy $b$ quark. The large samples of $b$ baryons collected by LHCb have led to greatly improved measurements of their lifetimes [9-12], which are in good agreement with HQET predictions. In particular, the lifetime of the $\Lambda_{b}^{0}$ baryon is now measured to a precision of better than $1 \%$ [13], and those of the $\Xi_{b}^{0}$ and $\Xi_{b}^{-}$to about $3 \%[12,13]$. Within HQET it is expected that the lifetimes of weakly decaying $b$ baryons follow the hierarchy $\tau_{\Omega_{b}^{-}} \simeq \tau_{\Xi_{b}^{-}}>\tau_{\Xi_{b}^{0}} \approx \tau_{\Lambda_{b}^{0}}[14-16]$, and thus far, the measured lifetimes respect this pattern within the uncertainties. However, the uncertainty on the measured lifetime of the $\Omega_{b}^{-}$baryon is too large to fully verify this prediction. The single best measurement to date of the $\Omega_{b}^{-}$lifetime is $1.54_{-0.21}^{+0.26} \pm 0.05$ ps [10] by the LHCb experiment, based on a sample of $58 \pm 8$ reconstructed $\Omega_{b}^{-} \rightarrow J / \psi \Omega^{-}$decays, with $J / \psi \rightarrow \mu^{+} \mu^{-}, \Omega^{-} \rightarrow \Lambda K^{-}$and $\Lambda \rightarrow p \pi^{-}$. Larger samples are needed to reduce the statistical uncertainty.

Improved knowledge of the $\Omega_{b}^{-}$mass would provide tighter experimental constraints for tests of lattice quantum chromodynamics (QCD) and QCD-inspired models, which aim to accurately predict the masses of hadrons [17]. The two most recent measurements of the $\Omega_{b}^{-}$mass, by the LHCb [18] and CDF [19] collaborations, are in agreement, but an earlier measurement by the D0 Collaboration [20] is larger by about 10 standard deviations.

*Full author list given at the end of the article.

Published by the American Physical Society under the terms of the Creative Commons Attribution 3.0 License. Further distribution of this work must maintain attribution to the author(s) and the published article's title, journal citation, and DOI.
In this paper, we report measurements of the mass and lifetime of the $\Omega_{b}^{-}$baryon using the decay mode $\Omega_{b}^{-} \rightarrow \Omega_{c}^{0} \pi^{-}$, where $\Omega_{c}^{0} \rightarrow p K^{-} K^{-} \pi^{+}$. (Charge-conjugate processes are implied throughout.) The only prior evidence of the $\Omega_{b}^{-} \rightarrow \Omega_{c}^{0} \pi^{-}$decay has been in the $\Omega_{c}^{0} \rightarrow \Omega^{-} \pi^{+}$ mode, with a signal of four events (3.3 $\sigma$ significance) [19]. The $\Omega_{c}^{0} \rightarrow p K^{-} K^{-} \pi^{+}$decay mode is Cabibbo-suppressed and is yet to be observed. However, it has the advantage of a larger acceptance in the $\mathrm{LHCb}$ detector compared to decay modes with hyperons in the final state. For example, the yield of $\Xi_{b}^{-}$decays reconstructed using $\Xi_{b}^{-} \rightarrow \Xi_{c}^{0} \pi^{-}, \Xi_{c}^{0} \rightarrow$ $p K^{-} K^{-} \pi^{+}$decays [12] is about 6 times larger than that obtained using $\Xi_{b}^{-} \rightarrow J / \psi \Xi^{-}$decays [10], where $\Xi^{-} \rightarrow$ $\Lambda \pi^{-}$and $\Lambda \rightarrow p \pi^{-}$.

The mass and lifetime measurements are calibrated with respect to those of the $\Xi_{b}^{-}$baryon, reconstructed in the $\Xi_{b}^{-} \rightarrow \Xi_{c}^{0} \pi^{-}, \Xi_{c}^{0} \rightarrow p K^{-} K^{-} \pi^{+}$decay mode. The mass and lifetime of the $\Xi_{b}^{-}$are measured to be $m_{\Xi_{b}^{-}}=5797.72 \pm$ $0.55 \mathrm{MeV} / c^{2}$ and $\tau_{\Xi_{b}^{-}}=1.599 \pm 0.041 \pm 0.022$ ps [12], respectively; the measurements are of sufficiently high precision that they do not represent a limiting uncertainty in the $\Omega_{b}^{-}$measurements presented here. The two quantities that are measured are the mass difference, $\delta m=m_{\Omega_{b}^{-}}-$ $m_{\Xi_{b}^{-}}$, and the lifetime ratio $\tau_{\Omega_{b}^{-}} / \tau_{\Xi_{b}^{-}}$. The identical final states and similar energy release in the $b$ - and $c$-baryon decays lead to a high degree of cancellation of the systematic uncertainties on these quantities. Throughout this article, we use $X_{b}\left(X_{c}\right)$ to refer to either a $\Xi_{b}^{-}\left(\Xi_{c}^{0}\right)$ or $\Omega_{b}^{-}\left(\Omega_{c}^{0}\right)$ baryon.

\section{DETECTOR AND SIMULATION}

The measurements use proton-proton $(p p)$ collision data samples, collected by the LHCb experiment, corresponding to an integrated luminosity of $3.0 \mathrm{fb}^{-1}$, of which $1.0 \mathrm{fb}^{-1}$ was recorded at a center-of-mass energy of $7 \mathrm{TeV}$ and $2.0 \mathrm{fb}^{-1}$ at $8 \mathrm{TeV}$. The LHCb detector [21,22] is a 
single-arm forward spectrometer covering the pseudorapidity range $2<\eta<5$, designed for the study of particles containing $b$ or $c$ quarks. The detector includes a highprecision tracking system consisting of a silicon-strip vertex detector surrounding the $p p$ interaction region, a large-area silicon-strip detector located upstream of a dipole magnet with a bending power of about $4 \mathrm{Tm}$, and three stations of silicon-strip detectors and straw drift tubes placed downstream of the magnet. The tracking system provides a measurement of momentum of charged particles with a relative uncertainty that varies from $0.5 \%$ at low momentum to $1.0 \%$ at $200 \mathrm{GeV} / c$. The minimum distance of a track to a primary vertex (PV), the impact parameter (IP), is measured with a resolution of $\left(15+29 / p_{\mathrm{T}}\right) \mu \mathrm{m}$, where $p_{\mathrm{T}}$ is the component of the momentum transverse to the beam, in $\mathrm{GeV} / c$. Different types of charged hadrons are distinguished using information from two ring-imaging Cherenkov detectors. Photons, electrons and hadrons are identified by a calorimeter system consisting of scintillating-pad and preshower detectors, an electromagnetic calorimeter and a hadronic calorimeter. Muons are identified by a system composed of alternating layers of iron and multiwire proportional chambers.

The online event selection is performed by a trigger [23], which consists of a hardware stage, based on information from the calorimeter and muon systems, followed by a software stage, which applies a full event reconstruction. The software trigger requires a two-, three- or four-track secondary vertex with a large $p_{\mathrm{T}}$ sum of the tracks and a significant displacement from the primary $p p$ interaction vertices. At least one particle should have $p_{\mathrm{T}}>1.7 \mathrm{GeV} / c$ and be inconsistent with coming from any of the PVs. The signal candidates are required to pass a multivariate software trigger selection algorithm [24].

Proton-proton collisions are simulated using PYTHIA [25] with a specific LHCb configuration [26]. Decays of hadronic particles are described by EvTGEN [27], in which final-state radiation is generated using Рнотоs [28]. The interaction of the generated particles with the detector, and its response, are implemented using the GEANT4 toolkit [29] as described in Ref. [30]. The $\Xi_{c}^{0} \rightarrow p K^{-} K^{-} \pi^{+}$and $\Omega_{c}^{0} \rightarrow p K^{-} K^{-} \pi^{+}$decays are modeled as an equal mixture of $X_{c} \rightarrow p K^{-} \bar{K}^{* 0}, \quad \bar{K}^{* 0} \rightarrow K^{-} \pi^{+}$and $X_{c} \rightarrow p K^{-} K^{-} \pi^{+}$ (nonresonant) decays; this composition reproduces well the only clear structure in these decays, a $\bar{K}^{* 0}$ peak in the $K^{-} \pi^{+}$mass distribution.

\section{CANDIDATE SELECTION}

Candidate $X_{c} \rightarrow p K^{-} K^{-} \pi^{+}$decays are formed by combining four tracks consistent with this decay chain and requiring a good quality vertex fit. In forming the $X_{c}$ candidate, each particle must be significantly detached from all $\mathrm{PVs}$ in the event, have $p_{\mathrm{T}}$ greater than $100 \mathrm{MeV} / c$, and have particle identification (PID) information consistent with the decay hypothesis. The PID requirements on the proton and the kaon candidates have a combined efficiency of $70 \%$ on signal, while reducing the combinatorial background by a factor of 3.5.

Candidate $X_{b}$ baryons are formed by combining an $X_{c}$ candidate with a $\pi^{-}$candidate. For each $X_{b}$ and PV pair in an event, a quantity $\chi_{\mathrm{IP}}^{2}\left(X_{b}\right)$ is computed, defined as the increase in $\chi^{2}$ when the $X_{b}$ candidate is included as an additional particle in the PV fit. The $X_{b}$ candidate is assigned to the PV with the smallest value of $\chi_{\mathrm{IP}}^{2}\left(X_{b}\right)$, and it is required to be significantly displaced from that PV. The invariant mass $M\left(p K^{-} K^{-} \pi^{+}\right)$is required to lie in the ranges $2461-2481 \mathrm{MeV} / c^{2}$ and $2685-2705 \mathrm{MeV} / c^{2}$ for $\Xi_{c}^{0}$ and $\Omega_{c}^{0}$ signal candidates, respectively; these intervals cover a mass region that represents about \pm 2.5 and \pm 2.0 times the expected mass resolution. The tighter requirement on the $\Omega_{c}^{0}$ candidates is used because of a lower signal-tobackground ratio. Candidates for which the $p K^{-} K^{-} \pi^{+}$ mass is outside the signal region are also used to model the $X_{c}$ combinatorial background contribution to the signal sample. To suppress the combinatorial background, candidate $X_{b}$ decays are required to have a reconstructed decay time larger than $0.2 \mathrm{ps}$, which is about 5 times the decaytime resolution for these decays.

To further improve the signal-to-background ratio, a multivariate analysis is employed, based on a boosted decision tree (BDT) algorithm [31,32] implemented within the TMVA package [33]. Simulated $\Xi_{b}^{-}$and $\Omega_{b}^{-}$decays are used to represent the signal distributions, and background events are taken from the signal sidebands in data. The sidebands consist of events that are close in mass to the $X_{b}$ signal region, but have either the $p K^{-} K^{-} \pi^{+}$or $X_{c} \pi^{-}$mass inconsistent with the known $X_{c}$ or $X_{b}$ masses. Independent training and test samples are used to ensure that the BDT is not overtrained.

A total of 18 discriminating variables are used to help differentiate signal and background candidates, including the $X_{b}$ decay vertex fit $\chi^{2}$; the $\chi_{\mathrm{IP}}^{2}$ of the $X_{b}, X_{c}$ and finalstate decay products; the consistency of the candidate with being produced at one of the PVs in the event; the $p_{\mathrm{T}}$ of the decay products; and the PID information on the proton and two kaons. Due to differences in the PID information between simulation and data, the distributions of PID variables for signal are taken from $D^{*+} \rightarrow D^{0} \pi^{+}$with $D^{0} \rightarrow K^{-} \pi^{+}, \Lambda \rightarrow p \pi^{-}$and $\Lambda_{c}^{+} \rightarrow p K^{-} \pi^{+}$decays in data [34], and are reweighted to account for differences in kinematics between the control and signal samples. The output of the training is a single discriminating variable that ranges from -1 to 1 . For convenience, the output value is also referred to as BDT.

The BDT requirement is chosen to maximize the figure of merit $N_{S} / \sqrt{N_{S}+N_{B}}$ for the $\Omega_{b}^{-}$signal. Here, $N_{S}$ and $N_{B}$ are the expected signal and background yields as a function of the BDT requirement. The chosen requirement of BDT $>0.3$ provides an expected signal (background) efficiency of about $90 \%(10 \%)$. 

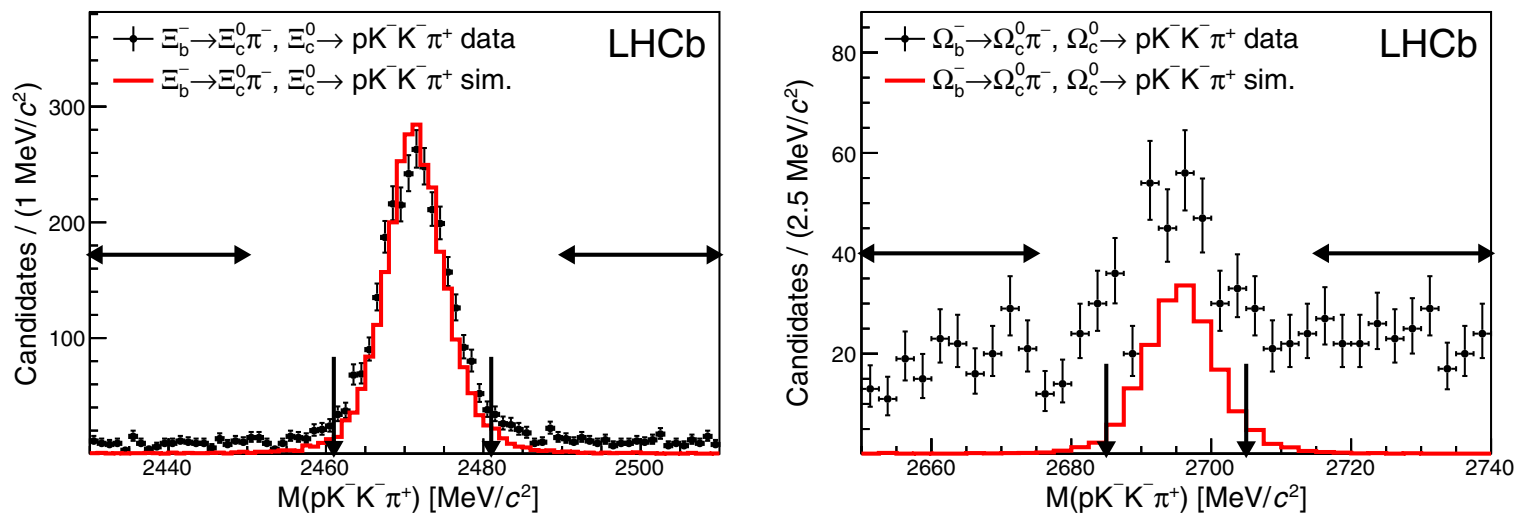

FIG. 1. Invariant mass distribution for (left) $\Xi_{c}^{0} \rightarrow p K^{-} K^{-} \pi^{+}$and (right) $\Omega_{c}^{0} \rightarrow p K^{-} K^{-} \pi^{+}$candidates over the full $X_{b}$ fit regions. The corresponding simulations (sim.) are overlaid. The vertical arrows indicate the signal regions, and the horizontal ones show the sideband regions.

\section{MASS SPECTRA AND FITS}

The $X_{c}$ invariant mass spectra for $X_{b}$ signal candidates are shown in Fig. 1. All candidates within the regions contributing to the $\Omega_{b}^{-}$mass fit, $5420-6380 \mathrm{MeV} / c^{2}$, and the $\Xi_{b}^{-}$mass fit, $5630-6590 \mathrm{MeV} / c^{2}$, are included. The simulated distributions, normalized to the fitted number of $X_{c}$ signal decays in data, are overlaid. The vertical and horizontal arrows indicate the signal and sideband regions.

While the overall background yields in these spectra are comparable, the signal-to-background ratio is much lower within the $\Omega_{c}^{0}$ candidate sample due to the lower production rate of $\Omega_{b}^{-}$relative to $\Xi_{b}^{-}$baryons, and likely a smaller $X_{c} \rightarrow$ $p K^{-} K^{-} \pi^{+}$branching fraction. Due to the very different $X_{c}$ background levels for the signal and calibration modes, we use the $X_{c}$ sidebands to model the $X_{c}$ combinatorial background in the $X_{b}$ invariant mass spectra.

To measure the $\Omega_{b}^{-}$mass and yield, the data are fitted using a simultaneous extended unbinned maximum likelihood fit to four $X_{b}$ invariant mass distributions; one pair is formed from the $X_{c}$ signal regions, and the second pair comprises events taken from the $X_{c}$ sidebands, as indicated in Fig. 1.

The signal shapes, determined from $\Omega_{b}^{-} \rightarrow \Omega_{c}^{0} \pi^{-}$and $\Xi_{b}^{-} \rightarrow \Xi_{c}^{0} \pi^{-}$simulated events, are each modeled by the sum of two Crystal Ball (CB) functions [35] which have a common mean value. The general forms of the two signal shapes are

$$
\begin{aligned}
\mathcal{F}_{\text {sig }}^{\Xi_{b}^{-}}= & f_{\text {low }} \mathrm{CB}_{-}\left(m_{0}, f_{\sigma} r_{\sigma} \sigma, \alpha_{-}, N_{-}\right) \\
& +\left(1-f_{\text {low }}\right) \mathrm{CB}_{+}\left(m_{0}, f_{\sigma} \sigma, \alpha_{+}, N_{+}\right), \\
\mathcal{F}_{\text {sig }}^{\Omega_{b}^{-}}= & f_{\text {low }} \mathrm{CB}_{-}\left(m_{0}+\delta m, r_{\sigma} \sigma, \alpha_{-}, N_{-}\right) \\
& +\left(1-f_{\text {low }}\right) \mathrm{CB}_{+}\left(m_{0}+\delta m, \sigma, \alpha_{+}, N_{+}\right) .
\end{aligned}
$$

Several of the parameters are common in the two signal shapes, and are determined from a simultaneous fit to the mass spectra from simulated samples of $\Omega_{b}^{-}$and $\Xi_{b}^{-}$decays. The $\mathrm{CB}_{ \pm}$function represents the signal contribution with a tail toward low (-) or high (+) invariant mass. The parameters $m_{0}$ and $m_{0}+\delta m$ represent the fitted peak mass values of the $\Xi_{b}^{-}$and $\Omega_{b}^{-}$baryons, respectively; $r_{\sigma}$ relates the lower $\mathrm{CB}$ width to the upper one, and $f_{\sigma}$ allows for a small difference in the mass resolution for the signal and calibration modes. The exponential tail parameters $\alpha_{ \pm}$are common to the signal and calibration modes. We fix the power-law tail parameters $N_{-}=N_{+}=10$, and the fraction $f_{\text {low }}=0.5$, as the simulated signal shapes are well described without these parameters freely varied. In fits to the data, $m_{0}, \delta m$ and $\sigma$ are left free to vary, and all other shape parameters are fixed to the values from the simulation.

Several sources of background contribute to the invariant mass spectrum for both the signal and the calibration modes. These include (i) partially reconstructed $X_{b} \rightarrow$ $X_{c} \rho^{-}$decays, (ii) misidentified $X_{b} \rightarrow X_{c} K^{-}$decays, (iii) partially reconstructed $\Omega_{b}^{-} \rightarrow \Omega_{c}^{* 0} \pi^{-}$decays ( $\Omega_{b}^{-}$only), (iv) random $X_{c} \rightarrow p K^{-} K^{-} \pi^{+}$combinations, and (v) the $X_{b} \rightarrow X_{c} \pi^{-}$combinatorial background. The $X_{b} \rightarrow X_{c} \rho^{-}$ background shape is based on simulated decays, and is parameterized by an ARGUS distribution [36] convolved with a Gaussian resolution function of $16.4 \mathrm{MeV} / c^{2}$ fixed width, the value obtained from fully reconstructed $\Omega_{b}^{-} \rightarrow$ $\Omega_{c}^{0} \pi^{-}$decays in data. The ARGUS shape parameters are left free to vary in the fit, as is the yield, expressed as a fraction of the $X_{b} \rightarrow X_{c} \pi^{-}$yield. The $X_{b} \rightarrow X_{c} K^{-}$background shape is fixed based on simulation. The yield fraction $N\left(X_{b} \rightarrow X_{c} K^{-}\right) / N\left(X_{b} \rightarrow X_{c} \pi^{-}\right)$is fixed to $3.1 \%$, which is the product of an assumed ratio of branching fractions $\mathcal{B}\left(X_{b} \rightarrow X_{c} K^{-}\right) / \mathcal{B}\left(X_{b} \rightarrow X_{c} \pi^{-}\right)=7 \%$, based on the value from $\Lambda_{b}^{0}$ decays [37], and the efficiency of the PID requirements on the $K^{-}$and $\pi^{-}$. The shape parameters used to describe these two backgrounds are common to the signal and calibration modes, apart from an overall mass offset, which is fixed to be equal to $\delta m$. The invariant mass distribution of the $\Omega_{b}^{-} \rightarrow \Omega_{c}^{* 0} \pi^{-}$background is taken from a 

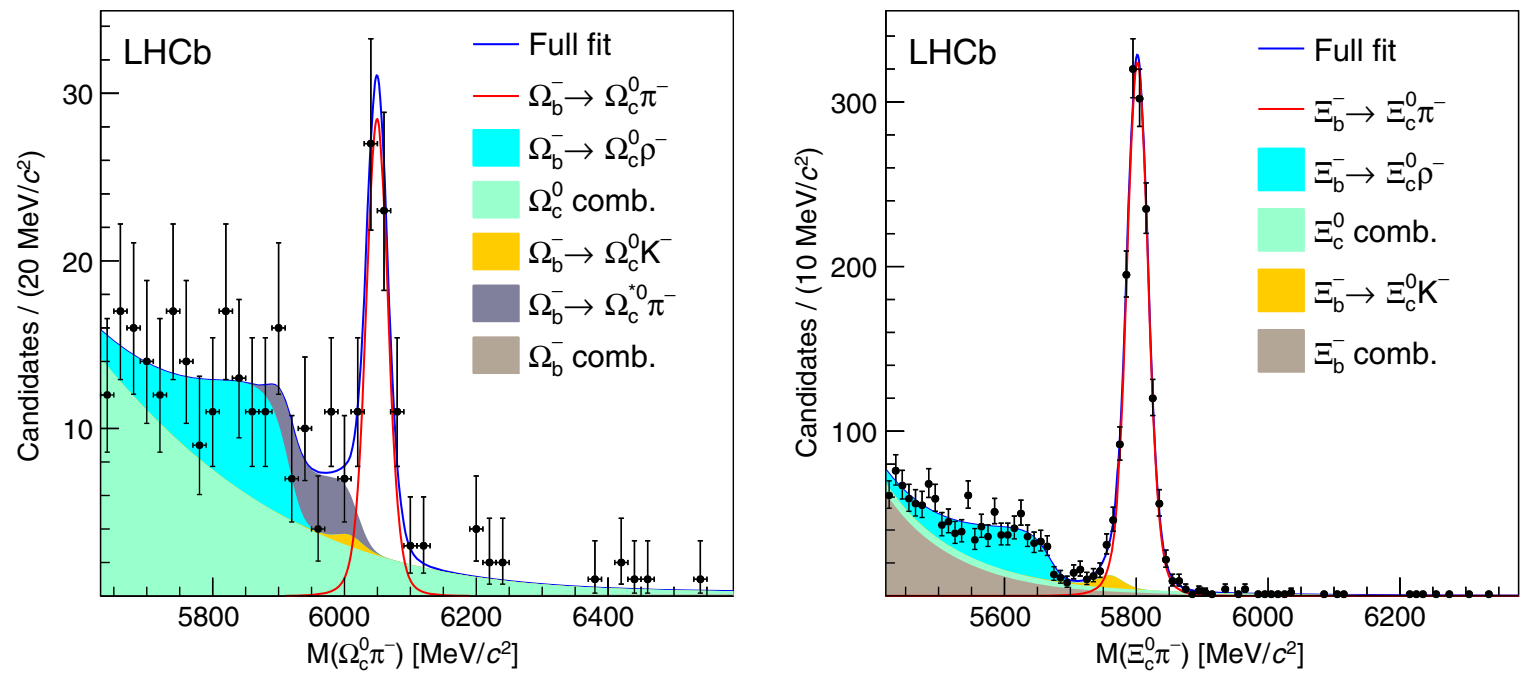

FIG. 2. Results of the simultaneous mass fit to the signal and calibration modes. The fitted $\Omega_{b}^{-}$combinatorial (comb.) background yield is very small, and not clearly visible.
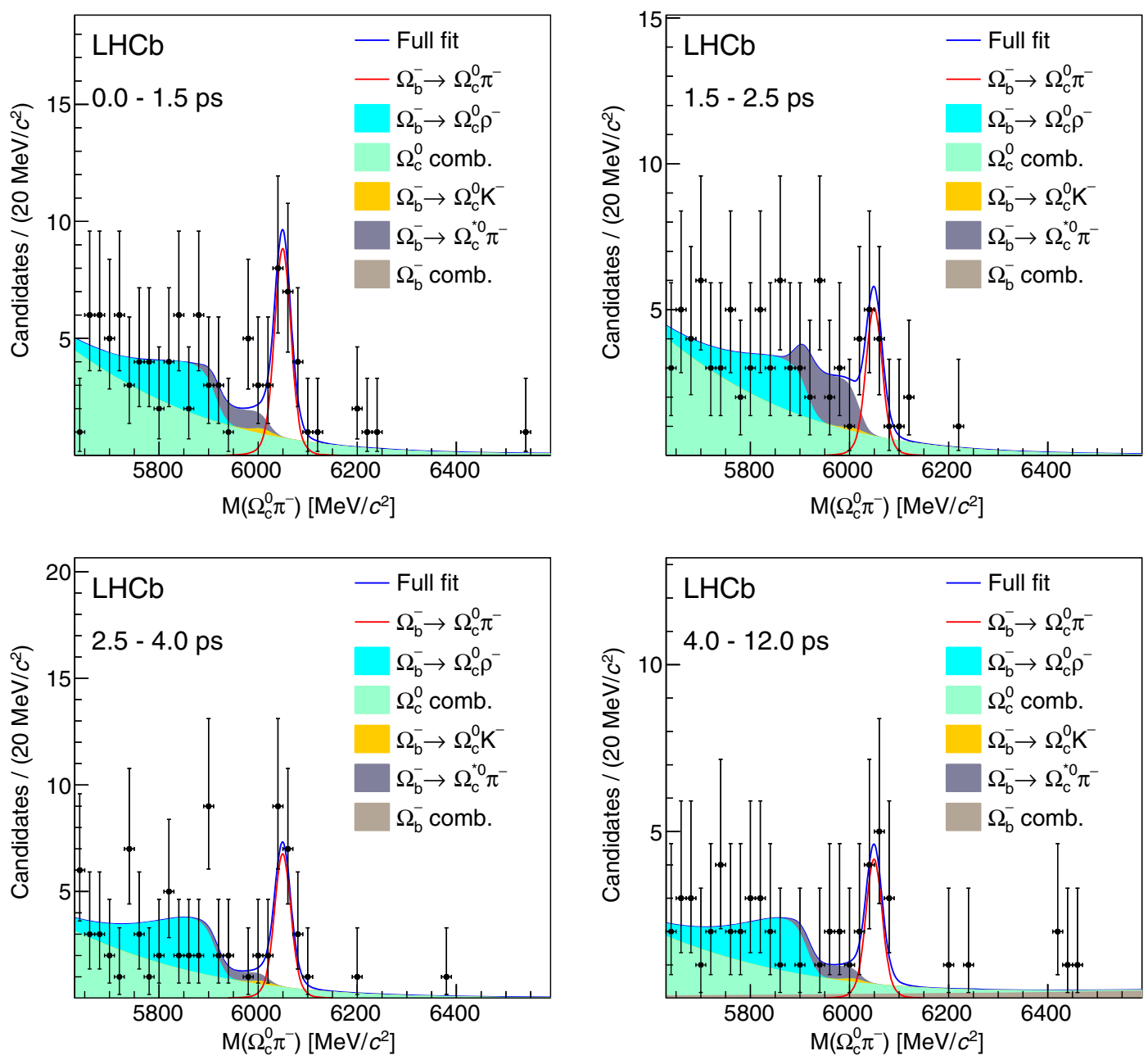

FIG. 3. Results of the simultaneous mass fit to the $\Omega_{b}^{-}$signal in the four decay-time bins, as indicated in each plot. 
parametrization of the mass distribution obtained from a phase-space simulation [38], combined with a Gaussian smearing based on the measured mass resolution. The yield fraction $N\left(\Omega_{b}^{-} \rightarrow \Omega_{c}^{0} \pi^{-}\right) / N\left(\Omega_{b}^{-} \rightarrow \Omega_{c}^{* 0} \pi^{-}\right)$is freely varied in the fit to data.

The $X_{c} \rightarrow p K^{-} K^{-} \pi^{+}$combinatorial background contribution is constrained by including the $X_{c}$ sidebands in the simultaneous fit, as discussed above. The shape of this background is modeled by the sum of a broad Gaussian function and an exponential shape. In the $X_{c}$ sidebands there is no indication of any $\Xi_{b}^{-}$or $\Omega_{b}^{-}$contributions, which might result from nonresonant $X_{b} \rightarrow p K^{-} K^{-} \pi^{+} \pi^{-}$decays. The shape parameters and yields of this background component are freely varied in the fit, but their values are common for the $X_{c}$ signal and sideband data samples. A different set of parameters is used for the $\Omega_{b}^{-}$and $\Xi_{b}^{-}$decay modes. The random $X_{c} \pi^{-}$combinatorial background is described by a single exponential function with variable slope and yield.

The $X_{b}$ invariant mass spectra with the fits overlaid are shown in Fig. 2 for the $X_{c}$ signal regions. The fitted yields are $62.6 \pm 9.0$ and $1384 \pm 39$ for the $\Omega_{b}^{-} \rightarrow \Omega_{c}^{0} \pi^{-}$and
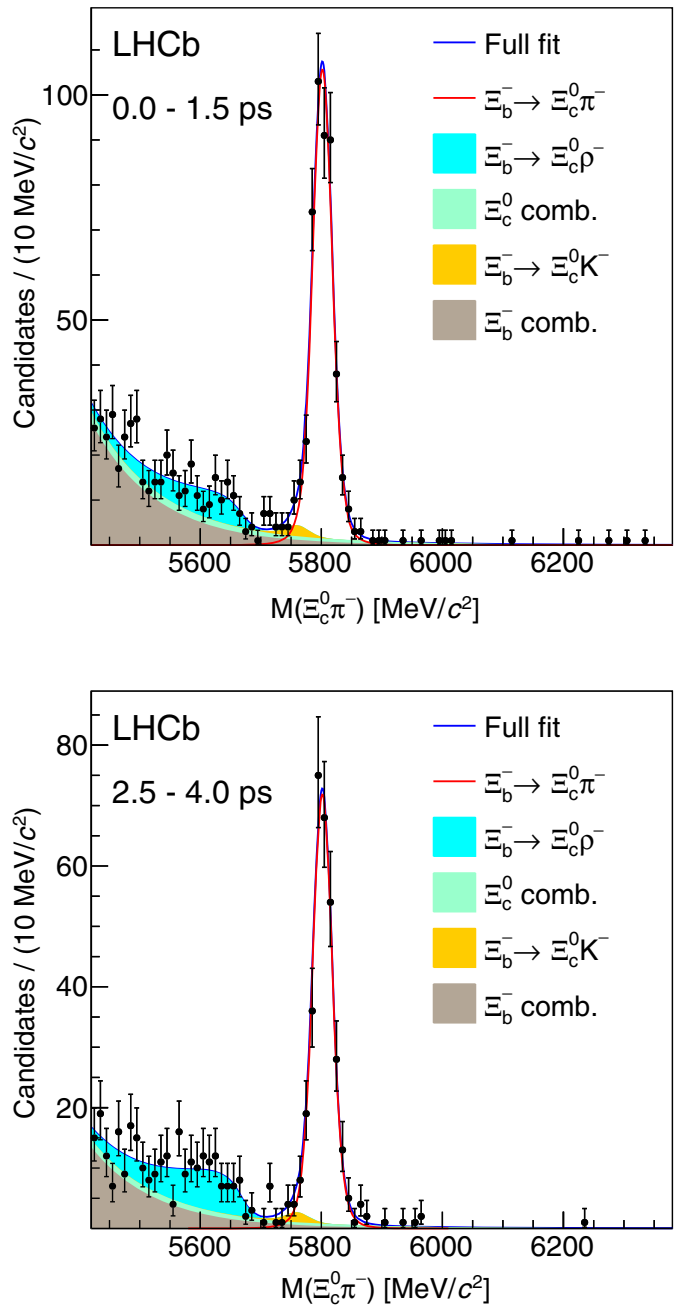

$\Xi_{b}^{-} \rightarrow \Xi_{c}^{0} \pi^{-}$modes, respectively. The $\Omega_{b}^{-} \rightarrow \Omega_{c}^{0} \pi^{-}, \Omega_{c}^{0} \rightarrow$ $p K^{-} K^{-} \pi^{+}$decay is observed for the first time with large significance, about 10 standard deviations based on Wilks's theorem [39]. The yield of $\Omega_{b}^{-} \rightarrow \Omega_{c}^{0} \pi^{-}$decays is comparable to that obtained in $\Omega_{b}^{-} \rightarrow J / \psi \Omega^{-}$decays [10]. The mass difference is measured to be $\delta m=247.7 \pm$ $3.0 \mathrm{MeV} / c^{2}$, where the uncertainty is statistical only.

\section{V. $\Omega_{b}^{-}$LIFETIME}

To measure the $\Omega_{b}^{-}$lifetime, the data from the signal and calibration modes are divided into four bins of $X_{b}$ decay time: $0.0-1.5 \mathrm{ps}, 1.5-2.5 \mathrm{ps}, 2.5-4.0 \mathrm{ps}$, and $4.0-12.0 \mathrm{ps}$. The decay-time binning was chosen based on pseudoexperiments which replicate the yields of events in data as a function of decay time for the signal and calibration modes. Several binning schemes were investigated, and the one above minimizes the systematic uncertainty on the lifetime due to the small $\Omega_{b}^{-}$sample size.

The yields in each decay-time bin in data are determined by repeating the mass fit for each decay-time bin, allowing
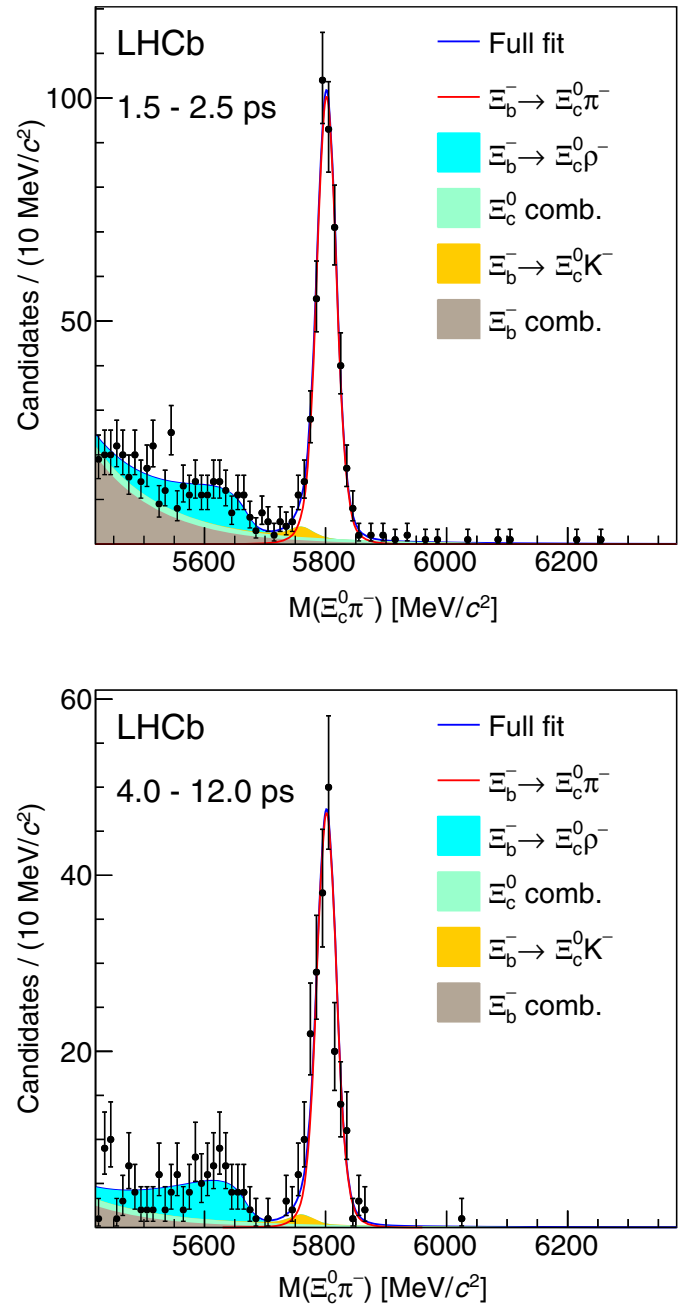

FIG. 4. Results of the simultaneous mass fit to the $\Xi_{b}^{-}$signal in the four decay-time bins, as indicated in each plot. 
TABLE I. Results of the fit to data for each decay-time bin, and the relative efficiency. The uncertainties are statistical only.

\begin{tabular}{lccc}
\hline \hline Decay-time bin $(\mathrm{ps})$ & $\Omega_{b}^{-}$yield & $\Xi_{b}^{-}$yield & $\epsilon\left(\Xi_{b}^{-}\right) / \epsilon\left(\Omega_{b}^{-}\right)$ \\
\hline $0.0-1.5$ & $20.8 \pm 4.8$ & $450 \pm 21$ & $1.10 \pm 0.03$ \\
$1.5-2.5$ & $12.0 \pm 3.7$ & $427 \pm 21$ & $1.11 \pm 0.04$ \\
$2.5-4.0$ & $17.7 \pm 4.2$ & $305 \pm 17$ & $1.02 \pm 0.04$ \\
$4.0-12.0$ & $10.5 \pm 3.3$ & $201 \pm 14$ & $1.03 \pm 0.05$ \\
\hline \hline
\end{tabular}

the signal and background yields to vary freely. All shape parameters are fixed to the values obtained from the fit to the whole data sample, since simulations show that they do not depend on the decay time. The results of the fits to the individual decay-time bins are shown in Figs. 3 and 4 for the signal and calibration modes. The yields are presented in Table I.

The relative efficiency in each bin is determined using simulated events. The efficiency-corrected yield ratio is then

$$
\frac{N_{\Omega_{b}^{-} \rightarrow \Omega_{c}^{0} \pi^{-}}(t)}{N_{\Xi_{b}^{-} \rightarrow \Xi^{0} \pi^{-}}(t)}=A \exp (\kappa t),
$$

where $A$ is a calibration factor, and

$$
\kappa \equiv 1 / \tau_{\Xi_{b}^{-}}-1 / \tau_{\Omega_{b}^{-}} .
$$

The value of $\kappa$ is obtained by fitting an exponential function to the efficiency-corrected ratio of yields, which in turn allows $\tau_{\Omega_{b}^{-}}$to be determined. The efficiencies for the signal and normalization modes are expressed as the fraction of generated signal decays with true decay time in bin $i$ which have a reconstructed decay time also in bin $i$. When defined in this way, effects of time resolution and selection requirements are accounted for, and the corrected signal and calibration mode yields are exponential in nature. The relative efficiencies after all selection requirements are given in Table I.

The efficiency ratio is consistent with having no dependence on the decay time, as expected from the similarity of the two decay modes. The efficiency-corrected yield ratio as a function of decay time is shown in Fig. 5, along with a $\chi^{2}$ fit to the data using an exponential function. The position of the points along the decay-time axis is determined by taking the average value within the bin, assuming an exponential decay-time distribution with $\tau=1.60 \mathrm{ps}$. From the fitted value of $\kappa=0.053 \pm 0.085 \mathrm{ps}^{-1}$ and the measured value of the $\Xi_{b}^{-}$lifetime, the lifetime ratio is found to be

$$
\frac{\tau_{\Omega_{b}^{-}}}{\tau_{\Xi_{b}^{-}}}=\frac{1}{1-\kappa \tau_{\Xi_{b}^{-}}}=1.09 \pm 0.16,
$$

where the uncertainty is statistical only.

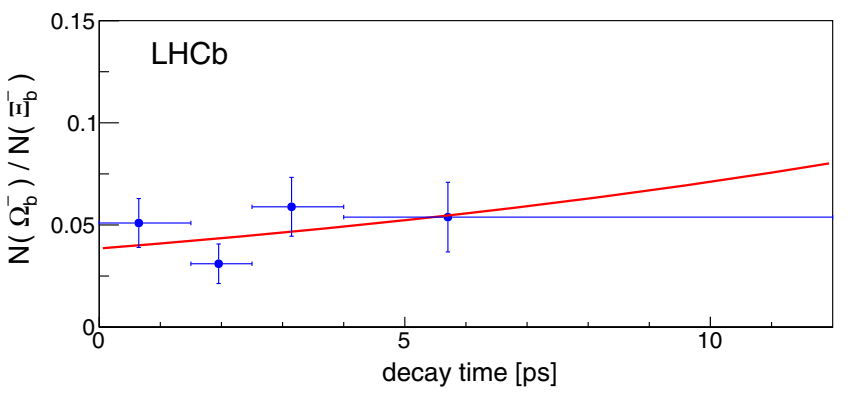

FIG. 5. Corrected signal yield ratio as a function of decay time, along with a fit to an exponential function. The horizontal bars indicate the bin sizes, and are not an indication of the uncertainty.

\section{SYSTEMATIC UNCERTAINTIES}

A number of systematic uncertainties are evaluated and summarized in Table II. Most of the systematic uncertainties are estimated by modifying each fixed input or function, and taking the difference with respect to the nominal value as the systematic uncertainty. The signal shape uncertainty is determined by changing the description to the sum of two Gaussian functions and repeating the analysis. The nominal $X_{c}$ combinatorial background shape is changed from the sum of a Gaussian shape and an exponential function to a single exponential distribution. The sensitivity to the $\Omega_{b}^{-} \rightarrow \Omega_{c}^{* 0} \pi^{-}$shape description is investigated by varying the shape parameters obtained from the simulation to account for the uncertainty on the mass resolution, as well as using a different function to parametrize the simulation. The uncertainty on the yield of misidentified $X_{b} \rightarrow X_{c} K^{-}$decays is quantified by varying the fractional contribution by $\pm 30 \%$ relative to the nominal value, to allow for uncertainty in the $X_{b} \rightarrow X_{c} K^{-}$branching fractions amongst these modes and for uncertainty in the PID efficiencies. The relative efficiency is obtained from simulation. However, the BDT performance in data is

TABLE II. Summary of systematic uncertainties in $\delta m$ and the lifetime ratio. When two values are indicated, the first is a correction, and the second is the uncertainty.

\begin{tabular}{lcc}
\hline \hline Source & $\delta m\left(\mathrm{MeV} / c^{2}\right)$ & $\tau_{\Omega_{b}^{-}} / \tau_{\Xi_{b}^{-}}$ \\
\hline Signal shape & \pm 0.3 & \pm 0.005 \\
Background shape & \pm 0.1 & \pm 0.009 \\
$\Omega_{c}^{* 0}$ shape & \pm 0.1 & \pm 0.003 \\
$X_{b} \rightarrow X_{c} K^{-}$background & \pm 0.2 & \pm 0.002 \\
Relative efficiency & $\ldots$ & \pm 0.018 \\
Average time in bin & $\ldots$ & \pm 0.002 \\
Lifetime fit & $\ldots$ & $+0.016 \pm 0.008$ \\
Simulated sample size & $-0.38 \pm 0.28$ & \pm 0.017 \\
Momentum scale & \pm 0.1 & $\ldots$ \\
$\Xi_{b}^{-}$lifetime & $\ldots$ & \pm 0.004 \\
Total systematic & $-0.4 \pm 0.5$ & $+0.016 \pm 0.029$ \\
Total statistical & \pm 3.2 & \pm 0.16 \\
\hline \hline
\end{tabular}


slightly worse than in simulation, so to estimate a potential bias in the lifetime ratio, we reevaluate the relative efficiency with a BDT $>0.6$ requirement, while keeping the nominal requirement on the data. This larger value was chosen since it provides equal efficiency of the BDT requirement on $\Xi_{b}^{-}$simulation and in data. To test the sensitivity to the position of the points along the decay-time axis (in Fig. 5), the fit is repeated assuming an exponential distribution with $\tau=1.80 \mathrm{ps}$. Bias due to the small signal size has been studied using pseudoexperiments, and we find a small fit bias in $\tau_{\Omega_{b}^{-}} / \tau_{\Xi_{b}^{-}}$, which pulls the value down by $10 \%$ of the statistical uncertainty. We correct the data for this bias, and assign half the shift as a systematic uncertainty. The simulated samples used to determine the relative efficiency are of finite size, and those uncertainties are propagated to the final result.

For the $\delta m$ measurement, the fitted value of $\delta m_{\text {meas }}-$ $\delta m_{\text {true }}$ in simulation is $-0.38 \pm 0.28 \mathrm{MeV} / c^{2}$. We apply this value as a correction, and assign the $0.28 \mathrm{MeV} / \mathrm{c}^{2}$ as a systematic uncertainty. The momentum scale has a fractional uncertainty of \pm 0.0003 [40]. Its effect is evaluated by shifting all momentum components of the final-state particles by this amount in simulated decays, and comparing to the case when no shift is applied. Lastly, the uncertainty in the $\Xi_{b}^{-}$lifetime enters weakly into the lifetime ratio [see Eq. (5)], and is also included as a source of uncertainty. All sources of systematic uncertainty are added in quadrature to obtain the corrections and systematic uncertainties of $-0.4 \pm 0.5 \mathrm{MeV} / c^{2}$ on $\delta m$ and $+0.016 \pm 0.029$ on $\tau_{\Omega_{b}^{-}} / \tau_{\Xi_{b}^{-}}$.

\section{SUMMARY}

In summary, a $3.0 \mathrm{fb}^{-1} p p$ collision data sample is used to reconstruct a sample of $63 \pm 9 \Omega_{b}^{-} \rightarrow \Omega_{c}^{0} \pi^{-}, \Omega_{c}^{0} \rightarrow$ $p K^{-} K^{-} \pi^{+}$decays. This is the first observation of these $\Omega_{b}^{-}$and $\Omega_{c}^{0}$ decay modes, with well over $5 \sigma$ significance. Using these signals, the mass difference and mass are measured to be

$$
\begin{aligned}
m_{\Omega_{b}^{-}}-m_{\Xi_{b}^{-}} & =247.3 \pm 3.2 \pm 0.5 \mathrm{MeV} / c^{2}, \\
m_{\Omega_{b}^{-}} & =6045.1 \pm 3.2 \pm 0.5 \pm 0.6 \mathrm{MeV} / c^{2},
\end{aligned}
$$

where the uncertainties are statistical, systematic, and from knowledge of the $\Xi_{b}^{-}$mass [12] ( $m_{\Omega_{b}^{-}}$only). The measured $\Omega_{b}^{-}$mass is consistent with previous measurements from $\mathrm{LHCb}, \quad 6046.0 \pm 2.2 \pm 0.5 \mathrm{MeV} / c^{2}$ [18], and $\mathrm{CDF}$, $6047.5 \pm 3.8 \pm 0.6 \mathrm{MeV} / c^{2}$ [19], but inconsistent with the value of $6165 \pm 10 \pm 13 \mathrm{MeV} / c^{2}$ obtained by the D0 experiment [20]. An average of the two LHCb measurements yields $m_{\Omega_{b}^{-}}=6045.7 \pm 1.9 \mathrm{MeV} / c^{2}$, where the momentum scale uncertainty is taken as $100 \%$ correlated, and the rest of the uncertainties are uncorrelated.
The lifetime ratio and absolute lifetime of the $\Omega_{b}^{-}$baryon are also measured to be

$$
\begin{aligned}
& \frac{\tau_{\Omega_{b}^{-}}}{\tau_{\Xi_{b}^{-}}}=1.11 \pm 0.16 \pm 0.03 \\
& \tau_{\Omega_{b}^{-}}=1.78 \pm 0.26 \pm 0.05 \pm 0.06 \mathrm{ps}
\end{aligned}
$$

using $\tau_{\Xi_{b}^{-}}=1.599 \pm 0.041 \pm 0.022 \mathrm{ps} \quad[12]$. The first uncertainty in each case is statistical. The second uncertainty on $\tau_{\Omega_{b}^{-}} / \tau_{\Xi_{b}^{-}}$is the total systematic uncertainty, as given in Table II. For $\tau_{\Omega_{b}^{-}}$, the second uncertainty is from all sources in Table II except the $\Xi_{b}^{-}$lifetime, and the third uncertainty stems from the uncertainty in the $\Xi_{b}^{-}$lifetime. The lifetime is consistent with the previous measurements of $\tau_{\Omega_{b}^{-}}=1.54_{-0.21}^{+0.26} \pm 0.05$ ps [10] and $\tau_{\Omega_{b}^{-}}=1.66_{-0.40}^{+0.53} \mathrm{ps}$ [19] by the $\mathrm{LHCb}$ and $\mathrm{CDF}$ collaborations, respectively. The average of the $\mathrm{LHCb}$ measurements, assuming no correlation among the uncertainties, yields an $\Omega_{b}^{-}$lifetime of $1.66_{-0.18}^{+0.19}$ ps. These measurements improve our knowledge of the mass and the lifetime of the $\Omega_{b}^{-}$baryon. Due to the similarity of the signal and calibration modes, this pair of decay modes is very promising for future studies of the $\Omega_{b}^{-}$baryon.

\section{ACKNOWLEDGMENTS}

We express our gratitude to our colleagues in the CERN accelerator departments for the excellent performance of the LHC. We thank the technical and administrative staff at the LHCb institutes. We acknowledge support from CERN and from the national agencies: CAPES, CNPq, FAPERJ and FINEP (Brazil); NSFC (China); CNRS/IN2P3 (France); BMBF, DFG and MPG (Germany); INFN (Italy); FOM and NWO (Netherlands); MNiSW and NCN (Poland); MEN/IFA (Romania); MinES and FANO (Russia); MinECo (Spain); SNSF and SER (Switzerland); NASU (Ukraine); STFC (United Kingdom); NSF (USA). We acknowledge the computing resources that are provided by CERN, IN2P3 (France), KIT and DESY (Germany), INFN (Italy), SURF (Netherlands), PIC (Spain), GridPP (United Kingdom), RRCKI and Yandex LLC (Russia), CSCS (Switzerland), IFIN-HH (Romania), CBPF (Brazil), PL-GRID (Poland) and OSC (USA). We are indebted to the communities behind the multiple open source software packages on which we depend. Individual groups or members have received support from the $\mathrm{AvH}$ Foundation (Germany); EPLANET, Marie SkłodowskaCurie Actions and ERC (European Union); Conseil Général de Haute-Savoie, Labex ENIGMASS and OCEVU, Région Auvergne (France); RFBR and Yandex LLC (Russia); GVA, XuntaGal and GENCAT (Spain); the Herchel Smith Fund, The Royal Society, Royal Commission for the Exhibition of 1851 and the Leverhulme Trust (United Kingdom). 
[1] V. A. Khoze and M. A. Shifman, Heavy quarks, Sov. Phys. Usp. 26, 387 (1983).

[2] I. I. Bigi and N. G. Uraltsev, Gluonic enhancements in nonspectator beauty decays-an inclusive mirage though an exclusive possibility, Phys. Lett. B 280, 271 (1992).

[3] I. I. Bigi, N. G. Uraltsev, and A. I. Vainshtein, Nonperturbative corrections to inclusive beauty and charm decays: QCD versus phenomenological models, Phys. Lett. B 293, 430 (1992); B297, 477(E) (1992).

[4] B. Blok and M. Shifman, The rule of discarding $1 / N_{c}$ in inclusive weak decays (I), Nucl. Phys. B399, 441 (1993).

[5] B. Blok and M. Shifman, The rule of discarding $1 / N_{c}$ in inclusive weak decays (II), Nucl. Phys. B399, 459 (1993).

[6] M. Neubert, B decays and the heavy quark expansion, Adv. Ser. Dir. High Energy Phys. 15, 239 (1998).

[7] N. Uraltsev, Heavy quark expansion in beauty and its decays, Proc. Int. Sch. Phys. Fermi 137, 329 (1998).

[8] G. Bellini, I. I. Y. Bigi, and P. J. Dornan, Lifetimes of charm and beauty hadrons, Phys. Rep. 289, 1 (1997).

[9] R. Aaij et al. (LHCb Collaboration), Precision measurement of the ratio of the $\Lambda_{b}^{0}$ to $\bar{B}^{0}$ lifetimes, Phys. Lett. B 734, 122 (2014).

[10] R. Aaij et al. (LHCb Collaboration), Measurement of the $\Xi_{b}^{-}$ and $\Omega_{b}^{-}$baryon lifetimes, Phys. Lett. B 736, 154 (2014).

[11] R. Aaij et al. (LHCb Collaboration), Precision measurement of the mass and lifetime of the $\Xi_{b}^{0}$ baryon, Phys. Rev. Lett. 113, 032001 (2014).

[12] R. Aaij et al. (LHCb Collaboration), Precision measurement of the mass and lifetime of the $\Xi_{b}^{-}$baryon, Phys. Rev. Lett. 113, 242002 (2014).

[13] K. A. Olive et al. (Particle Data Group Collaboration), Review of particle physics, Chin. Phys. C 38, 090001 (2014), and 2015 update.

[14] I. I. Y. Bigi, The QCD perspective on lifetimes of heavy flavor hadrons, arXiv:hep-ph/9508408.

[15] H.-Y. Cheng, A phenomenological analysis of heavy hadron lifetimes, Phys. Rev. D 56, 2783 (1997).

[16] T. Ito, M. Matsuda, and Y. Matsui, New possibility of solving the problem of lifetime ratio $\tau\left(\Lambda_{b}^{0}\right) / \tau\left(B_{d}\right)$, Prog. Theor. Phys. 99, 271 (1998).

[17] C. Amsler, T. Degrand, and B. Krusche, Quark model, published in Ref. [13].

[18] R. Aaij et al. (LHCb Collaboration), Measurements of the $\Lambda_{b}^{0}, \Xi_{b}^{-}$, and $\Omega_{b}^{-}$baryon masses, Phys. Rev. Lett. 110, 182001 (2013).

[19] T. A. Aaltonen et al. (CDF Collaboration), Mass and lifetime measurements of bottom and charm baryons in $p \bar{p}$ collisions at $\sqrt{s}=1.96 \mathrm{TeV}$, Phys. Rev. D 89, 072014 (2014).

[20] V. M. Abazov et al. (D0 Collaboration), Observation of the doubly strange $b$ baryon $\Omega_{b}^{-}$, Phys. Rev. Lett. 101, 232002 (2008).

[21] A. A. Alves, Jr. et al. (LHCb Collaboration), The LHCb detector at the LHC, J. Instrum. 3, S08005 (2008).

[22] R. Aaij et al. (LHCb Collaboration), LHCb detector performance, Int. J. Mod. Phys. A 30, 1530022 (2015).
[23] R. Aaij et al., The LHCb trigger and its performance in 2011, J. Instrum. 8, P04022 (2013).

[24] V. V. Gligorov and M. Williams, Efficient, reliable and fast high-level triggering using a bonsai boosted decision tree, J. Instrum. 8, P02013 (2013).

[25] T. Sjöstrand, S. Mrenna, and P. Skands, PYTHIA 6.4 physics and manual, J. High Energy Phys. 05 (2006) 026; A brief introduction to PYTHIA 8.1, Comput. Phys. Commun. 178, 852 (2008).

[26] I. Belyaev et al., Handling of the generation of primary events in Gauss, the LHCb simulation framework, J. Phys. Conf. Ser. 331, 032047 (2011).

[27] D. J. Lange, The EvtGen particle decay simulation package, Nucl. Instrum. Methods Phys. Res., Sect. A 462, 152 (2001).

[28] P. Golonka and Z. Was, PHOTOS Monte Carlo: A precision tool for QED corrections in $Z$ and $W$ decays, Eur. Phys. J. C 45, 97 (2006).

[29] J. Allison et al. (Geant4 Collaboration), Geant4 developments and applications, IEEE Trans. Nucl. Sci. 53, 270 (2006); S. Agostinelli et al. (Geant4 Collaboration), Geant4: A simulation toolkit, Nucl. Instrum. Methods Phys. Res., Sect. A 506, 250 (2003).

[30] M. Clemencic, G. Corti, S. Easo, C. R. Jones, S. Miglioranzi, M. Pappagallo, and P. Robbe, The LHCb simulation application, Gauss: Design, evolution and experience, J. Phys. Conf. Ser. 331, 032023 (2011).

[31] L. Breiman, J. H. Friedman, R. A. Olshen, and C. J. Stone, Classification and Regression Trees (Wadsworth, Belmont, CA, 1984).

[32] R. E. Schapire and Y. Freund, A decision-theoretic generalization of on-line learning and an application to boosting, J. Comput. Syst. Sci. 55, 119 (1997).

[33] A. Hoecker et al., TMVA: Toolkit for multivariate data analysis, Proc. Sci., ACAT2007 (2007) 040.

[34] M. Adinolfi et al., Performance of the LHCb RICH detector at the LHC, Eur. Phys. J. C 73, 2431 (2013).

[35] T. Skwarnicki, Ph.D. thesis, Institute of Nuclear Physics, Krakow, 1986, DESY-F31-86-02.

[36] H. Albrecht et al. (ARGUS Collaboration), Measurement of the polarization in the decay $B \rightarrow J / \psi K^{*}$, Phys. Lett. B340, 217 (1994).

[37] R. Aaij et al. (LHCb Collaboration), Study of beauty baryon decays to $D^{0} p h^{-}$and $\Lambda_{c}^{+} h^{-}$final states, Phys. Rev. D 89, 032001 (2014).

[38] R. Brun and F. Rademakers, ROOT: An object oriented data analysis framework, Nucl. Instrum. Methods Phys. Res., Sect. A 389, 81 (1997); see https://root.cern.ch/ doc/master/classTGenPhaseSpace.html for additional details.

[39] S. S. Wilks, The large-sample distribution of the likelihood ratio for testing composite hypotheses, Ann. Math. Stat. 9, 60 (1938).

[40] R. Aaij et al. (LHCb Collaboration), Precision measurement of $D$ meson mass differences, J. High Energy Phys. 06 (2013) 065. 
R. Aaij, ${ }^{39}$ C. Abellán Beteta, ${ }^{41}$ B. Adeva, ${ }^{38}$ M. Adinolfi, ${ }^{47}$ Z. Ajaltouni, ${ }^{5}$ S. Akar, ${ }^{6}$ J. Albrecht, ${ }^{10}$ F. Alessio, ${ }^{39}$ M. Alexander, ${ }^{52}$ S. Ali, ${ }^{42}$ G. Alkhazov, ${ }^{31}$ P. Alvarez Cartelle, ${ }^{54}$ A. A. Alves Jr., ${ }^{58}$ S. Amato, ${ }^{2}$ S. Amerio, ${ }^{23}$ Y. Amhis, ${ }^{7}$ L. An,${ }^{3,40}$ L. Anderlini, ${ }^{18}$ G. Andreassi, ${ }^{40}$ M. Andreotti, ${ }^{17, a}$ J. E. Andrews, ${ }^{59}$ R. B. Appleby, ${ }^{55}$ O. Aquines Gutierrez, ${ }^{11}$ F. Archilli, ${ }^{39}$ P. d'Argent, ${ }^{12}$ A. Artamonov, ${ }^{36}$ M. Artuso,${ }^{60}$ E. Aslanides, ${ }^{6}$ G. Auriemma, ${ }^{26, b}$ M. Baalouch, ${ }^{5}$ S. Bachmann, ${ }^{12}$ J. J. Back, ${ }^{49}$ A. Badalov, ${ }^{37}$ C. Baesso, ${ }^{61}$ S. Baker, ${ }^{54}$ W. Baldini, ${ }^{17}$ R. J. Barlow,${ }^{55}$ C. Barschel,${ }^{39}$ S. Barsuk, ${ }^{7}$ W. Barter,${ }^{39}$ V. Batozskaya, ${ }^{29}$ V. Battista, ${ }^{40}$ A. Bay,${ }^{40}$ L. Beaucourt, ${ }^{4}$ J. Beddow,${ }^{52}$ F. Bedeschi, ${ }^{24}$ I. Bediaga, ${ }^{1}$ L. J. Bel,${ }^{42}$ V. Bellee,${ }^{40}$

N. Belloli, ${ }^{21, c}$ I. Belyaev, ${ }^{32}$ E. Ben-Haim, ${ }^{8}$ G. Bencivenni, ${ }^{19}$ S. Benson, ${ }^{39}$ J. Benton, ${ }^{47}$ A. Berezhnoy,${ }^{33}$ R. Bernet,${ }^{41}$ A. Bertolin, ${ }^{23}$ F. Betti, ${ }^{15}$ M.-O. Bettler, ${ }^{39}$ M. van Beuzekom, ${ }^{42}$ S. Bifani, ${ }^{46}$ P. Billoir, ${ }^{8}$ T. Bird, ${ }^{55}$ A. Birnkraut, ${ }^{10}$ A. Bizzeti, ${ }^{18, d}$ T. Blake, ${ }^{49}$ F. Blanc, ${ }^{40}$ J. Blouw, ${ }^{11}$ S. Blusk,${ }^{60}$ V. Bocci ${ }^{26}$ A. Bondar, ${ }^{35}$ N. Bondar, ${ }^{31,39}$ W. Bonivento, ${ }^{16}$ A. Borgheresi,${ }^{21, c}$ S. Borghi, ${ }^{55}$ M. Borisyak, ${ }^{67}$ M. Borsato, ${ }^{38}$ M. Boubdir, ${ }^{9}$ T. J. V. Bowcock, ${ }^{53}$ E. Bowen, ${ }^{41}$ C. Bozzi,,${ }^{17,39}$ S. Braun, ${ }^{12}$

M. Britsch,${ }^{12}$ T. Britton, ${ }^{60}$ J. Brodzicka, ${ }^{55}$ E. Buchanan, ${ }^{47}$ C. Burr, ${ }^{55}$ A. Bursche, ${ }^{2}$ J. Buytaert, ${ }^{39}$ S. Cadeddu, ${ }^{16}$ R. Calabrese, ${ }^{17, a}$ M. Calvi, ${ }^{21, c}$ M. Calvo Gomez, ${ }^{37, \mathrm{e}}$ P. Campana, ${ }^{19}$ D. Campora Perez, ${ }^{39}$ L. Capriotti, ${ }^{55}$ A. Carbone,,${ }^{15, f}$ G. Carboni, ${ }^{25, \mathrm{~g}}$ R. Cardinale, ${ }^{20, \mathrm{~h}}$ A. Cardini, ${ }^{16}$ P. Carniti, ${ }^{21, \mathrm{c}}$ L. Carson, ${ }^{51}$ K. Carvalho Akiba, ${ }^{2}$ G. Casse,${ }^{53}$ L. Cassina, ${ }^{21, \mathrm{c}}$ L. Castillo Garcia, ${ }^{40}$ M. Cattaneo, ${ }^{39}$ Ch. Cauet, ${ }^{10}$ G. Cavallero, ${ }^{20}$ R. Cenci, ${ }^{24, i}$ M. Charles, ${ }^{8}$ Ph. Charpentier, ${ }^{39}$ G. Chatzikonstantinidis, ${ }^{46}$ M. Chefdeville, ${ }^{4}$ S. Chen,${ }^{55}$ S.-F. Cheung, ${ }^{56}$ V. Chobanova, ${ }^{38}$ M. Chrzaszcz, ${ }^{41,27}$ X. Cid Vidal, ${ }^{39}$ G. Ciezarek, ${ }^{42}$ P.E. L. Clarke, ${ }^{51}$ M. Clemencic, ${ }^{39}$ H. V. Cliff, ${ }^{48}$ J. Closier, ${ }^{39}$ V. Coco, ${ }^{58}$ J. Cogan, ${ }^{6}$ E. Cogneras, ${ }^{5}$ V. Cogoni, ${ }^{16, j}$ L. Cojocariu, ${ }^{30}$ G. Collazuol, ${ }^{23, k}$ P. Collins, ${ }^{39}$ A. Comerma-Montells, ${ }^{12}$ A. Contu, ${ }^{39}$ A. Cook,${ }^{47}$ S. Coquereau, ${ }^{8}$ G. Corti, ${ }^{39}$ M. Corvo, ${ }^{17, a}$ B. Couturier, ${ }^{39}$ G. A. Cowan, ${ }^{51}$ D. C. Craik,${ }^{51}$ A. Crocombe, ${ }^{49}$ M. Cruz Torres, ${ }^{61}$ S. Cunliffe, ${ }^{54}$ R. Currie ${ }^{54}$ C. D’Ambrosio, ${ }^{39}$ E. Dall'Occo, ${ }^{42}$ J. Dalseno, ${ }^{47}$ P. N. Y. David,${ }^{42}$ A. Davis, ${ }^{58}$ O. De Aguiar Francisco, ${ }^{2}$ K. De Bruyn, ${ }^{6}$ S. De Capua, ${ }^{55}$ M. De Cian, ${ }^{12}$ J. M. De Miranda, ${ }^{1}$ L. De Paula, ${ }^{2}$ P. De Simone, ${ }^{19}$ C.-T. Dean, ${ }^{52}$ D. Decamp, ${ }^{4}$ M. Deckenhoff, ${ }^{10}$ L. Del Buono, ${ }^{8}$ N. Déléage, ${ }^{4}$ M. Demmer, ${ }^{10}$ D. Derkach,${ }^{67}$ O. Deschamps, ${ }^{5}$ F. Dettori, ${ }^{39}$ B. Dey, ${ }^{22}$ A. Di Canto, ${ }^{39}$ H. Dijkstra, ${ }^{39}$ F. Dordei,${ }^{39}$ M. Dorigo, ${ }^{40}$ A. Dosil Suárez, ${ }^{38}$ A. Dovbnya, ${ }^{44}$ K. Dreimanis,${ }^{53}$ L. Dufour, ${ }^{42}$ G. Dujany, ${ }^{55}$ K. Dungs, ${ }^{39}$ P. Durante,${ }^{39}$ R. Dzhelyadin, ${ }^{36}$ A. Dziurda,${ }^{27}$ A. Dzyuba,${ }^{31}$ S. Easo,${ }^{50,39}$ U. Egede, ${ }^{54}$ V. Egorychev, ${ }^{32}$ S. Eidelman, ${ }^{35}$ S. Eisenhardt,${ }^{51}$ U. Eitschberger, ${ }^{10}$ R. Ekelhof, ${ }^{10}$ L. Eklund, ${ }^{52}$ I. El Rifai, ${ }^{5}$ Ch. Elsasser ${ }^{41}$ S. Ely, ${ }^{60}$ S. Esen, ${ }^{12}$ H. M. Evans, ${ }^{48}$ T. Evans, ${ }^{56}$ A. Falabella, ${ }^{15}$ C. Färber,${ }^{39}$ N. Farley, ${ }^{46}$ S. Farry, ${ }^{53}$ R. Fay, ${ }^{53}$ D. Fazzini, ${ }^{21, c}$ D. Ferguson, ${ }^{51}$ V. Fernandez Albor ${ }^{38}$ F. Ferrari, ${ }^{15}$ F. Ferreira Rodrigues, ${ }^{1}$ M. Ferro-Luzzi, ${ }^{39}$ S. Filippov, ${ }^{34}$ M. Fiore, ${ }^{17, a}$ M. Fiorini, ${ }^{17, a}$ M. Firlej ${ }^{28}$ C. Fitzpatrick,${ }^{40}$ T. Fiutowski, ${ }^{28}$ F. Fleuret,${ }^{7,1}$ K. Fohl,${ }^{39}$ M. Fontana, ${ }^{16}$ F. Fontanelli, ${ }^{20, h}$ D. C. Forshaw, ${ }^{60}$ R. Forty, ${ }^{39}$ M. Frank,${ }^{39}$ C. Frei, ${ }^{39}$ M. Frosini, ${ }^{18}$ J. Fu, ${ }^{22}$ E. Furfaro, ${ }^{25, g}$ A. Gallas Torreira, ${ }^{38}$ D. Galli,,${ }^{15, f}$ S. Gallorini, ${ }^{23}$ S. Gambetta, ${ }^{51}$ M. Gandelman, ${ }^{2}$ P. Gandini,${ }^{56}$ Y. Gao, ${ }^{3}$ J. García Pardiñas, ${ }^{38}$ J. Garra Tico, ${ }^{48}$ L. Garrido,${ }^{37}$ P. J. Garsed, ${ }^{48}$ D. Gascon, ${ }^{37}$ C. Gaspar, ${ }^{39}$ L. Gavardi, ${ }^{10}$ G. Gazzoni, ${ }^{5}$ D. Gerick, ${ }^{12}$ E. Gersabeck, ${ }^{12}$ M. Gersabeck,${ }^{55}$ T. Gershon, ${ }^{49} \mathrm{Ph}$. Ghez, ${ }^{4}$ S. Gianì, ${ }^{40}$ V. Gibson, ${ }^{48}$ O. G. Girard,${ }^{40}$ L. Giubega, ${ }^{30}$ V. V. Gligorov ${ }^{39}$ C. Göbel,${ }^{61}$ D. Golubkov, ${ }^{32}$ A. Golutvin, ${ }^{54,39}$ A. Gomes, ${ }^{1, \mathrm{~m}}$ C. Gotti, ${ }^{21, \mathrm{c}}$ M. Grabalosa Gándara, ${ }^{5}$ R. Graciani Diaz, ${ }^{37}$ L. A. Granado Cardoso, ${ }^{39}$ E. Graugés, ${ }^{37}$ E. Graverini, ${ }^{41}$ G. Graziani, ${ }^{18}$ A. Grecu, ${ }^{30}$ P. Griffith, ${ }^{46}$ L. Grillo, ${ }^{12}$ O. Grünberg, ${ }^{65}$ E. Gushchin,${ }^{34}$ Yu. Guz,${ }^{36,39}$ T. Gys, ${ }^{39}$ T. Hadavizadeh,${ }^{56}$ C. Hadjivasiliou, ${ }^{60}$ G. Haefeli ${ }^{40}$ C. Haen, ${ }^{39}$ S. C. Haines, ${ }^{48}$ S. Hall,${ }^{54}$ B. Hamilton, ${ }^{59}$ X. Han, ${ }^{12}$ S. Hansmann-Menzemer, ${ }^{12}$ N. Harnew, ${ }^{56}$ S. T. Harnew, ${ }^{47}$ J. Harrison, ${ }^{55}$ J. He, ${ }^{39}$ T. Head, ${ }^{40}$ A. Heister, ${ }^{9}$ K. Hennessy, ${ }^{53}$ P. Henrard, ${ }^{5}$ L. Henry, ${ }^{8}$ J. A. Hernando Morata, ${ }^{38}$ E. van Herwijnen, ${ }^{39}$ M. Heß ${ }^{65}$ A. Hicheur, ${ }^{2}$ D. Hill, ${ }^{56}$ M. Hoballah, C. Hombach, ${ }^{55}$ L. Hongming, ${ }^{40}$ W. Hulsbergen, ${ }^{42}$ T. Humair, ${ }^{54}$ M. Hushchyn,${ }^{67}$ N. Hussain,${ }^{56}$ D. Hutchcroft,${ }^{53}$ M. Idzik, ${ }^{28}$ P. Ilten, ${ }^{57}$ R. Jacobsson, ${ }^{39}$ A. Jaeger, ${ }^{12}$ J. Jalocha, ${ }^{56}$ E. Jans, ${ }^{42}$ A. Jawahery, ${ }^{59}$ M. John, ${ }^{56}$ D. Johnson, ${ }^{39}$ C. R. Jones,${ }^{48}$ C. Joram, ${ }^{39}$ B. Jost, ${ }^{39}$ N. Jurik ${ }^{60}$ S. Kandybei,${ }^{44}$ W. Kanso, ${ }^{6}$ M. Karacson, ${ }^{39}$ T. M. Karbach, ${ }^{39, \dagger}$ S. Karodia, ${ }^{52}$ M. Kecke, ${ }^{12}$ M. Kelsey, ${ }^{60}$ I. R. Kenyon, ${ }^{46}$ M. Kenzie, ${ }^{39}$ T. Ketel, ${ }^{43}$ E. Khairullin, ${ }^{67}$ B. Khanji, ${ }^{21,39, c}$ C. Khurewathanakul, ${ }^{40}$ T. Kirn, ${ }^{9}$ S. Klaver, ${ }^{55}$ K. Klimaszewski, ${ }^{29}$ M. Kolpin, ${ }^{12}$ I. Komarov,${ }^{40}$ R. F. Koopman, ${ }^{43}$ P. Koppenburg, ${ }^{42}$ M. Kozeiha, ${ }^{5}$ L. Kravchuk, ${ }^{34}$ K. Kreplin, ${ }^{12}$ M. Kreps,${ }^{49}$ P. Krokovny, ${ }^{35}$ F. Kruse, ${ }^{10}$ W. Krzemien, ${ }^{29}$ W. Kucewicz, ${ }^{27, n}$ M. Kucharczyk,${ }^{27}$ V. Kudryavtsev, ${ }^{35}$ A. K. Kuonen, ${ }^{40}$ K. Kurek, ${ }^{29}$ T. Kvaratskheliya, ${ }^{32}$ D. Lacarrere, ${ }^{39}$ G. Lafferty, ${ }^{55,39}$ A. Lai, ${ }^{16}$ D. Lambert, ${ }^{51}$ G. Lanfranchi, ${ }^{19}$ C. Langenbruch, ${ }^{49}$ B. Langhans, ${ }^{39}$ T. Latham, ${ }^{49}$ C. Lazzeroni, ${ }^{46}$ R. Le Gac, ${ }^{6}$ J. van Leerdam, ${ }^{42}$ J.-P. Lees, ${ }^{4}$ R. Lefèvre, ${ }^{5}$ A. Leflat, ${ }^{33,39}$ J. Lefrançois, ${ }^{7}$ E. Lemos Cid, ${ }^{38}$ O. Leroy, ${ }^{6}$ T. Lesiak, ${ }^{27}$ B. Leverington, ${ }^{12}$ Y. Li, $^{7}$ T. Likhomanenko, ${ }^{67,66}$ R. Lindner, ${ }^{39}$ C. Linn,${ }^{39}$ F. Lionetto, ${ }^{41}$ B. Liu, ${ }^{16}$ X. Liu, ${ }^{3}$ D. Loh, ${ }^{49}$ I. Longstaff, ${ }^{52}$ J. H. Lopes, ${ }^{2}$ D. Lucchesi, ${ }^{23, \mathrm{k}}$ M. Lucio Martinez, ${ }^{38}$ H. Luo, ${ }^{51}$ A. Lupato, ${ }^{23}$ E. Luppi,${ }^{17, a}$ O. Lupton, ${ }^{56}$ N. Lusardi, ${ }^{22}$ A. Lusiani, ${ }^{24}$ X. Lyu, ${ }^{62}$ F. Machefert, ${ }^{7}$ F. Maciuc,${ }^{30}$ O. Maev,${ }^{31}$ K. Maguire,${ }^{55}$ S. Malde,${ }^{56}$ A. Malinin,${ }^{66}$ G. Manca, ${ }^{7}$ G. Mancinelli, ${ }^{6}$ P. Manning,${ }^{60}$ A. Mapelli, ${ }^{39}$ J. Maratas, ${ }^{5}$ J. F. Marchand, ${ }^{4}$ U. Marconi, ${ }^{15}$ C. Marin Benito, ${ }^{37}$ P. Marino, ${ }^{24, i}$ J. Marks, ${ }^{12}$ G. Martellotti, ${ }^{26}$ 
M. Martin, ${ }^{6}$ M. Martinelli, ${ }^{40}$ D. Martinez Santos,${ }^{38}$ F. Martinez Vidal ${ }^{68}$ D. Martins Tostes, ${ }^{2}$ L. M. Massacrier, ${ }^{7}$ A. Massafferri, ${ }^{1}$ R. Matev ${ }^{39}$ A. Mathad,${ }^{49}$ Z. Mathe,${ }^{39}$ C. Matteuzzi, ${ }^{21}$ A. Mauri, ${ }^{41}$ B. Maurin,${ }^{40}$ A. Mazurov, ${ }^{46}$ M. McCann,${ }^{54}$ J. McCarthy, ${ }^{46}$ A. McNab,${ }^{55}$ R. McNulty, ${ }^{13}$ B. Meadows,${ }^{58}$ F. Meier, ${ }^{10}$ M. Meissner, ${ }^{12}$ D. Melnychuk, ${ }^{29}$ M. Merk, ${ }^{42}$ A Merli, ${ }^{22,0}$ E Michielin, ${ }^{23}$ D. A. Milanes, ${ }^{64}$ M.-N. Minard, ${ }^{4}$ D. S. Mitzel,,${ }^{12}$ J. Molina Rodriguez, ${ }^{61}$ I. A. Monroy, ${ }^{64}$ S. Monteil, ${ }^{5}$ M. Morandin, ${ }^{23}$ P. Morawski, ${ }^{28}$ A. Mordà, ${ }^{6}$ M. J. Morello, ${ }^{24, i}$ J. Moron, ${ }^{28}$ A. B. Morris, ${ }^{51}$ R. Mountain, ${ }^{60}$ F. Muheim, ${ }^{51}$ D. Müller ${ }^{55}$ J. Müller,${ }^{10}$ K. Müller ${ }^{41}$ V. Müller, ${ }^{10}$ M. Mussini ${ }^{15}$ B. Muster ${ }^{40}$ P. Naik, ${ }^{47}$ T. Nakada, ${ }^{40}$ R. Nandakumar, ${ }^{50}$ A. Nandi,${ }^{56}$ I. Nasteva, ${ }^{2}$ M. Needham,${ }^{51}$ N. Neri, ${ }^{22}$ S. Neubert, ${ }^{12}$ N. Neufeld, ${ }^{39}$ M. Neuner, ${ }^{12}$ A. D. Nguyen, ${ }^{40}$ C. Nguyen-Mau, ${ }^{40, p}$ V. Niess, ${ }^{5}$ S. Nieswand,,${ }^{9}$ R. Niet, ${ }^{10}$ N. Nikitin, ${ }^{33}$ T. Nikodem, ${ }^{12}$ A. Novoselov, ${ }^{36}$

D. P. O'Hanlon, ${ }^{49}$ A. Oblakowska-Mucha, ${ }^{28}$ V. Obraztsov, ${ }^{36}$ S. Ogilvy, ${ }^{52}$ O. Okhrimenko, ${ }^{45}$ R. Oldeman, ${ }^{16,48, j}$ C. J. G. Onderwater, ${ }^{69}$ B. Osorio Rodrigues, ${ }^{1}$ J. M. Otalora Goicochea, ${ }^{2}$ A. Otto, ${ }^{39}$ P. Owen, ${ }^{54}$ A. Oyanguren ${ }^{68}$ A. Palano,${ }^{14, q}$ F. Palombo, ${ }^{22,0}$ M. Palutan, ${ }^{19}$ J. Panman, ${ }^{39}$ A. Papanestis,${ }^{50}$ M. Pappagallo, ${ }^{52}$ L. L. Pappalardo, ${ }^{17, a}$ C. Pappenheimer, ${ }^{58}$ W. Parker, ${ }^{59}$ C. Parkes,${ }^{55}$ G. Passaleva, ${ }^{18}$ G. D. Patel,${ }^{53}$ M. Patel,${ }^{54}$ C. Patrignani,,${ }^{20, h}$ A. Pearce,${ }^{55,50}$ A. Pellegrino, ${ }^{42}$ G. Penso ${ }^{26, r}$ M. Pepe Altarelli, ${ }^{39}$ S. Perazzini,,${ }^{15, f}$ P. Perret,${ }^{5}$ L. Pescatore ${ }^{46}$ K. Petridis, ${ }^{47}$ A. Petrolini,${ }^{20, h}$ M. Petruzzo, ${ }^{22}$ E. Picatoste Olloqui, ${ }^{37}$ B. Pietrzyk, ${ }^{4}$ M. Pikies, ${ }^{27}$ D. Pinci, ${ }^{26}$ A. Pistone, ${ }^{20}$ A. Piucci, ${ }^{12}$ S. Playfer, ${ }^{51}$ M. Plo Casasus, ${ }^{38}$ T. Poikela,${ }^{39}$ F. Polci, ${ }^{8}$ A. Poluektov, ${ }^{49,35}$ I. Polyakov, ${ }^{32}$ E. Polycarpo, ${ }^{2}$ A. Popov ${ }^{36}$ D. Popov,,${ }^{11,39}$ B. Popovici, ${ }^{30}$ C. Potterat, ${ }^{2}$ E. Price, ${ }^{47}$ J. D. Price, ${ }^{53}$ J. Prisciandaro, ${ }^{38}$ A. Pritchard,${ }^{53}$ C. Prouve, ${ }^{47}$ V. Pugatch,${ }^{45}$ A. Puig Navarro, ${ }^{40}$ G. Punzi, ${ }^{24, s}$ W. Qian, ${ }^{56}$ R. Quagliani, ${ }^{7,47}$ B. Rachwal, ${ }^{27}$ J. H. Rademacker, ${ }^{47}$ M. Rama, ${ }^{24}$ M. Ramos Pernas, ${ }^{38}$ M. S. Rangel, ${ }^{2}$ I. Raniuk, ${ }^{44}$ G. Raven, ${ }^{43}$ F. Redi, ${ }^{54}$ S. Reichert, ${ }^{10}$ A. C. dos Reis, ${ }^{1}$ V. Renaudin, ${ }^{7}$ S. Ricciardi, ${ }^{50}$ S. Richards,${ }^{47}$ M. Rihl,${ }^{39}$ K. Rinnert,${ }^{53,39}$ V. Rives Molina, ${ }^{37}$ P. Robbe, ${ }^{7}$ A. B. Rodrigues, ${ }^{1}$ E. Rodrigues,${ }^{58}$ J. A. Rodriguez Lopez, ${ }^{64}$ P. Rodriguez Perez, ${ }^{55}$ A. Rogozhnikov, ${ }^{67}$ S. Roiser, ${ }^{39}$ V. Romanovsky, ${ }^{36}$ A. Romero Vidal, ${ }^{38}$ J. W. Ronayne, ${ }^{13}$ M. Rotondo, ${ }^{23}$ T. Ruf, ${ }^{39}$ P. Ruiz Valls, ${ }^{68}$ J. J. Saborido Silva, ${ }^{38}$ N. Sagidova, ${ }^{31}$ B. Saitta, ${ }^{16, j}$

V. Salustino Guimaraes, ${ }^{2}$ C. Sanchez Mayordomo, ${ }^{68}$ B. Sanmartin Sedes,${ }^{38}$ R. Santacesaria, ${ }^{26}$ C. Santamarina Rios, ${ }^{38}$ M. Santimaria, ${ }^{19}$ E. Santovetti, ${ }^{25,8}$ A. Sarti, ${ }^{19, \mathrm{r}}$ C. Satriano, ${ }^{26, \mathrm{~b}}$ A. Satta, ${ }^{25}$ D. M. Saunders, ${ }^{47}$ D. Savrina,${ }^{32,33}$ S. Schael, ${ }^{9}$ M. Schiller, ${ }^{39}$ H. Schindler, ${ }^{39}$ M. Schlupp, ${ }^{10}$ M. Schmelling, ${ }^{11}$ T. Schmelzer, ${ }^{10}$ B. Schmidt, ${ }^{39}$ O. Schneider, ${ }^{40}$ A. Schopper,${ }^{39}$ M. Schubiger, ${ }^{40}$ M.-H. Schune, ${ }^{7}$ R. Schwemmer, ${ }^{39}$ B. Sciascia, ${ }^{19}$ A. Sciubba,${ }^{26, r}$ A. Semennikov, ${ }^{32}$ A. Sergi, ${ }^{46}$ N. Serra, ${ }^{41}$ J. Serrano, ${ }^{6}$ L. Sestini, ${ }^{23}$ P. Seyfert, ${ }^{21}$ M. Shapkin, ${ }^{36}$ I. Shapoval, ${ }^{17,44, a}$ Y. Shcheglov, ${ }^{31}$ T. Shears, ${ }^{53}$ L. Shekhtman, ${ }^{35}$ V. Shevchenko, ${ }^{66}$ A. Shires, ${ }^{10}$ B. G. Siddi, ${ }^{17}$ R. Silva Coutinho, ${ }^{41}$ L. Silva de Oliveira, ${ }^{2}$ G. Simi, ${ }^{23, s}$ M. Sirendi, ${ }^{48}$ N. Skidmore, ${ }^{47}$ T. Skwarnicki, ${ }^{60}$ E. Smith, ${ }^{54}$ I. T. Smith, ${ }^{51}$ J. Smith, ${ }^{48}$ M. Smith, ${ }^{55}$ H. Snoek, ${ }^{42}$ M. D. Sokoloff, ${ }^{58}$ F. J. P. Soler, ${ }^{52}$ F. Soomro, ${ }^{40}$ D. Souza, ${ }^{47}$ B. Souza De Paula, ${ }^{2}$ B. Spaan,${ }^{10}$ P. Spradlin,${ }^{52}$ S. Sridharan, ${ }^{39}$ F. Stagni, ${ }^{39}$ M. Stahl,${ }^{12}$ S. Stahl,${ }^{39}$ S. Stefkova, ${ }^{54}$ O. Steinkamp, ${ }^{41}$ O. Stenyakin, ${ }^{36}$ S. Stevenson,${ }^{56}$ S. Stoica,${ }^{30}$ S. Stone,${ }^{60}$ B. Storaci, ${ }^{41}$ S. Stracka, ${ }^{24, i}$ M. Straticiuc, ${ }^{30}$ U. Straumann, ${ }^{41}$ L. Sun ${ }^{58}$ W. Sutcliffe, ${ }^{54}$ K. Swientek, ${ }^{28}$ S. Swientek, ${ }^{10}$ V. Syropoulos,${ }^{43}$ M. Szczekowski, ${ }^{29}$ T. Szumlak ${ }^{28}$ S. T'Jampens, ${ }^{4}$ A. Tayduganov, ${ }^{6}$ T. Tekampe, ${ }^{10}$ G. Tellarini, ${ }^{17, a}$ F. Teubert,${ }^{39}$ C. Thomas, ${ }^{56}$ E. Thomas, ${ }^{39}$ J. van Tilburg, ${ }^{42}$ V. Tisserand, ${ }^{4} \mathrm{M}$. Tobin,${ }^{40} \mathrm{~S}$. Tolk, ${ }^{43}$ L. Tomassetti, ${ }^{17, a}$ D. Tonelli, ${ }^{39}$ S. Topp-Joergensen, ${ }^{56}$ E. Tournefier, ${ }^{4}$ S. Tourneur, ${ }^{40}$ K. Trabelsi, ${ }^{40}$ M. Traill, ${ }^{52}$ M. T. Tran, ${ }^{40}$ M. Tresch, ${ }^{41}$ A. Trisovic, ${ }^{39}$ A. Tsaregorodtsev, ${ }^{6}$ P. Tsopelas, ${ }^{42}$ N. Tuning, ${ }^{42,39}$ A. Ukleja, ${ }^{29}$ A. Ustyuzhanin, ${ }^{67,66}$ U. Uwer, ${ }^{12}$ C. Vacca,,${ }^{16,39, j}$ V. Vagnoni,${ }^{15,39}$ S. Valat, ${ }^{39}$ G. Valenti, ${ }^{15}$ A. Vallier ${ }^{7}$ R. Vazquez Gomez, ${ }^{19}$ P. Vazquez Regueiro, ${ }^{38}$ C. Vázquez Sierra, ${ }^{38}$ S. Vecchi, ${ }^{17}$ M. van Veghel, ${ }^{42}$ J. J. Velthuis, ${ }^{47}$ M. Veltri, ${ }^{18, t}$ G. Veneziano, ${ }^{40}$ M. Vesterinen, ${ }^{12}$ B. Viaud, ${ }^{7}$ D. Vieira, ${ }^{2}$ M. Vieites Diaz, ${ }^{38}$

X. Vilasis-Cardona, ${ }^{37, e}$ V. Volkov, ${ }^{33}$ A. Vollhardt ${ }^{41}$ D. Voong, ${ }^{47}$ A. Vorobyev, ${ }^{31}$ V. Vorobyev, ${ }^{35}$ C. Voß,${ }^{65}$ J. A. de Vries,${ }^{42}$ R. Waldi, ${ }^{65}$ C. Wallace, ${ }^{49}$ R. Wallace, ${ }^{13}$ J. Walsh,${ }^{24}$ J. Wang, ${ }^{60}$ D. R. Ward, ${ }^{48}$ N. K. Watson, ${ }^{46}$ D. Websdale,${ }^{54}$ A. Weiden, ${ }^{41}$ M. Whitehead, ${ }^{39}$ J. Wicht, ${ }^{49}$ G. Wilkinson, ${ }^{56,39}$ M. Wilkinson, ${ }^{60}$ M. Williams,${ }^{39}$ M. P. Williams, ${ }^{46}$ M. Williams, ${ }^{57}$ T. Williams, ${ }^{46}$ F. F. Wilson, ${ }^{50}$ J. Wimberley, ${ }^{59}$ J. Wishahi, ${ }^{10}$ W. Wislicki, ${ }^{29}$ M. Witek, ${ }^{27}$ G. Wormser, ${ }^{7}$ S. A. Wotton, ${ }^{48}$ K. Wraight, ${ }^{52}$ S. Wright, ${ }^{48}$ K. Wyllie, ${ }^{39}$ Y. Xie, ${ }^{63}$ Z. Xu, ${ }^{40}$ Z. Yang, ${ }^{3}$ H. Yin,${ }^{63}$ J. Yu, ${ }^{63}$ X. Yuan, ${ }^{35}$ O. Yushchenko, ${ }^{36}$ M. Zangoli, ${ }^{15}$ M. Zavertyaev, ${ }^{11, u}$ L. Zhang, ${ }^{3}$ Y. Zhang, ${ }^{3}$ A. Zhelezov, ${ }^{12}$ Y. Zheng, ${ }^{62}$ A. Zhokhov, ${ }^{32}$ L. Zhong, ${ }^{3}$ V. Zhukov, ${ }^{9}$ and S. Zucchelli ${ }^{15}$

\section{(LHCb Collaboration)}

${ }^{1}$ Centro Brasileiro de Pesquisas Físicas (CBPF), Rio de Janeiro, Brazil

${ }^{2}$ Universidade Federal do Rio de Janeiro (UFRJ), Rio de Janeiro, Brazil

${ }^{3}$ Center for High Energy Physics, Tsinghua University, Beijing, China 
${ }^{4}$ LAPP, Université Savoie Mont-Blanc, CNRS/IN2P3, Annecy-Le-Vieux, France

${ }^{5}$ Clermont Université, Université Blaise Pascal, CNRS/IN2P3, LPC, Clermont-Ferrand, France

${ }^{6}$ CPPM, Aix-Marseille Université, CNRS/IN2P3, Marseille, France

${ }^{7}$ LAL, Université Paris-Sud, CNRS/IN2P3, Orsay, France

${ }^{8}$ LPNHE, Université Pierre et Marie Curie, Université Paris Diderot, CNRS/IN2P3, Paris, France

${ }^{9}$ I. Physikalisches Institut, RWTH Aachen University, Aachen, Germany

${ }^{10}$ Fakultät Physik, Technische Universität Dortmund, Dortmund, Germany

${ }^{11}$ Max-Planck-Institut für Kernphysik (MPIK), Heidelberg, Germany

${ }^{12}$ Physikalisches Institut, Ruprecht-Karls-Universität Heidelberg, Heidelberg, Germany

${ }^{13}$ School of Physics, University College Dublin, Dublin, Ireland

${ }^{14}$ Sezione INFN di Bari, Bari, Italy

${ }^{15}$ Sezione INFN di Bologna, Bologna, Italy

${ }^{16}$ Sezione INFN di Cagliari, Cagliari, Italy

${ }^{17}$ Sezione INFN di Ferrara, Ferrara, Italy

${ }^{18}$ Sezione INFN di Firenze, Firenze, Italy

${ }^{19}$ Laboratori Nazionali dell'INFN di Frascati, Frascati, Italy

${ }^{20}$ Sezione INFN di Genova, Genova, Italy

${ }^{21}$ Sezione INFN di Milano Bicocca, Milano, Italy

${ }^{22}$ Sezione INFN di Milano, Milano, Italy

${ }^{23}$ Sezione INFN di Padova, Padova, Italy

${ }^{24}$ Sezione INFN di Pisa, Pisa, Italy

${ }^{25}$ Sezione INFN di Roma Tor Vergata, Roma, Italy

${ }^{26}$ Sezione INFN di Roma La Sapienza, Roma, Italy

${ }^{27}$ Henryk Niewodniczanski Institute of Nuclear Physics Polish Academy of Sciences, Kraków, Poland

${ }^{28}$ AGH-University of Science and Technology,

Faculty of Physics and Applied Computer Science, Kraków, Poland

${ }^{29}$ National Center for Nuclear Research (NCBJ), Warsaw, Poland

${ }^{30}$ Horia Hulubei National Institute of Physics and Nuclear Engineering, Bucharest-Magurele, Romania

${ }^{31}$ Petersburg Nuclear Physics Institute (PNPI), Gatchina, Russia

${ }^{32}$ Institute of Theoretical and Experimental Physics (ITEP), Moscow, Russia

${ }^{33}$ Institute of Nuclear Physics, Moscow State University (SINP MSU), Moscow, Russia

${ }^{34}$ Institute for Nuclear Research of the Russian Academy of Sciences (INR RAN), Moscow, Russia

${ }^{35}$ Budker Institute of Nuclear Physics (SB RAS) and Novosibirsk State University, Novosibirsk, Russia

${ }^{36}$ Institute for High Energy Physics (IHEP), Protvino, Russia

${ }^{37}$ Universitat de Barcelona, Barcelona, Spain

${ }^{38}$ Universidad de Santiago de Compostela, Santiago de Compostela, Spain

${ }^{39}$ European Organization for Nuclear Research (CERN), Geneva, Switzerland

${ }^{40}$ Ecole Polytechnique Fédérale de Lausanne (EPFL), Lausanne, Switzerland

${ }^{41}$ Physik-Institut, Universität Zürich, Zürich, Switzerland

${ }^{42}$ Nikhef National Institute for Subatomic Physics, Amsterdam, The Netherlands

${ }^{43}$ Nikhef National Institute for Subatomic Physics and VU University Amsterdam, Amsterdam, The Netherlands

${ }^{44}$ NSC Kharkiv Institute of Physics and Technology (NSC KIPT), Kharkiv, Ukraine

${ }^{45}$ Institute for Nuclear Research of the National Academy of Sciences (KINR), Kyiv, Ukraine

${ }^{46}$ University of Birmingham, Birmingham, United Kingdom

${ }^{47}$ H. H. Wills Physics Laboratory, University of Bristol, Bristol, United Kingdom

${ }^{48}$ Cavendish Laboratory, University of Cambridge, Cambridge, United Kingdom

${ }^{49}$ Department of Physics, University of Warwick, Coventry, United Kingdom

${ }^{50}$ STFC Rutherford Appleton Laboratory, Didcot, United Kingdom

${ }^{51}$ School of Physics and Astronomy, University of Edinburgh, Edinburgh, United Kingdom

${ }^{52}$ School of Physics and Astronomy, University of Glasgow, Glasgow, United Kingdom

${ }^{53}$ Oliver Lodge Laboratory, University of Liverpool, Liverpool, United Kingdom

${ }^{54}$ Imperial College London, London, United Kingdom

${ }^{55}$ School of Physics and Astronomy, University of Manchester, Manchester, United Kingdom

${ }^{56}$ Department of Physics, University of Oxford, Oxford, United Kingdom

${ }^{57}$ Massachusetts Institute of Technology, Cambridge, Massachusetts, USA

${ }^{58}$ University of Cincinnati, Cincinnati, Ohio, USA

${ }^{59}$ University of Maryland, College Park, Maryland, USA

${ }^{60}$ Syracuse University, Syracuse, New York, USA 
${ }^{61}$ Pontifícia Universidade Católica do Rio de Janeiro (PUC-Rio),

Rio de Janeiro, Brazil (associated with Institution Universidade Federal do Rio de Janeiro (UFRJ), Rio de Janeiro, Brazil)

${ }^{62}$ University of Chinese Academy of Sciences, Beijing, China (associated with Institution Center for High Energy Physics, Tsinghua University, Beijing, China)

${ }^{63}$ Institute of Particle Physics, Central China Normal University, Wuhan, Hubei, China (associated with Institution Center for High Energy Physics, Tsinghua University, Beijing, China)

${ }^{64}$ Departamento de Fisica, Universidad Nacional de Colombia,

Bogota, Colombia (associated with Institution LPNHE, Université Pierre et Marie Curie, Université Paris Diderot, CNRS/IN2P3, Paris, France)

${ }^{65}$ Institut für Physik, Universität Rostock, Rostock, Germany (associated with Institution Physikalisches Institut, Ruprecht-Karls-Universität Heidelberg, Heidelberg, Germany)

${ }^{66}$ National Research Centre Kurchatov Institute, Moscow, Russia (associated with Institution Institute of Theoretical and Experimental Physics (ITEP), Moscow, Russia)

${ }^{67}$ Yandex School of Data Analysis, Moscow, Russia (associated with Institution Institute of Theoretical and Experimental Physics (ITEP), Moscow, Russia)

${ }^{68}$ Instituto de Fisica Corpuscular (IFIC), Universitat de Valencia-CSIC, Valencia, Spain (associated with Institution Universitat de Barcelona, Barcelona, Spain)

${ }^{69}$ Van Swinderen Institute, University of Groningen, Groningen, The Netherlands (associated with Institution Nikhef National Institute for Subatomic Physics, Amsterdam, The Netherlands)

${ }^{\dagger}$ Deceased.

${ }^{\mathrm{a}}$ Also at Università di Ferrara, Ferrara, Italy.

${ }^{\mathrm{b}}$ Also at Università della Basilicata, Potenza, Italy.

${ }^{\mathrm{c}}$ Also at Università di Milano Bicocca, Milano, Italy.

${ }^{\mathrm{d}}$ Also at Università di Modena e Reggio Emilia, Modena, Italy.

eAlso at LIFAELS, La Salle, Universitat Ramon Llull, Barcelona, Spain.

${ }^{\mathrm{f}}$ Also at Università di Bologna, Bologna, Italy.

${ }^{g}$ Also at Università di Roma Tor Vergata, Roma, Italy.

${ }^{\mathrm{h}}$ Also at Università di Genova, Genova, Italy.

${ }^{\mathrm{i}}$ Also at Scuola Normale Superiore, Pisa, Italy.

${ }^{\mathrm{j}}$ Also at Università di Cagliari, Cagliari, Italy.

${ }^{\mathrm{k}}$ Also at Università di Padova, Padova, Italy.

${ }^{1}$ Also at Laboratoire Leprince-Ringuet, Palaiseau, France.

${ }^{\mathrm{m}}$ Also at Universidade Federal do Triângulo Mineiro (UFTM), Uberaba-MG, Brazil.

${ }^{\mathrm{n}}$ Also at AGH-University of Science and Technology, Faculty of Computer Science, Electronics and Telecommunications, Kraków, Poland.

${ }^{\circ}$ Also at Università degli Studi di Milano, Milano, Italy.

${ }^{\mathrm{p}}$ Also at Hanoi University of Science, Hanoi, Viet Nam.

${ }^{\mathrm{q}}$ Also at Università di Bari, Bari, Italy.

${ }^{\mathrm{r}}$ Also at Università di Roma La Sapienza, Roma, Italy.

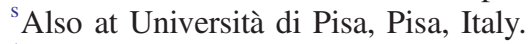

${ }^{\mathrm{t}}$ Also at Università di Urbino, Urbino, Italy

uAlso at P.N. Lebedev Physical Institute, Russian Academy of Science (LPI RAS), Moscow, Russia. 